Journal of Nepal Mathematical Society (JNMS), Vol. 1, Issue 2 (2018); D.Panthi

\title{
A Meir-Keeler Type Common Fixed Point Result in Dislocated Metric Space
}

\author{
Dinesh Panthi \\ Department of Mathematics, Valmeeki Campus \\ Nepal Sanskrit University, Kathmandu, Nepal. \\ Correspondence to: Dinesh Panthi, Email: panthid06@gmail.com
}

\begin{abstract}
A. Meir and E. Keeler [11] generalized the Banach Contraction Principle [1] with the notion of weakly uniformly strict contraction which is famous as a $(\varepsilon-\delta)$ contraction. In this article, we establish a Meir- Keeler type common fixed point result in dislocated metric space which generalize and extend similar fixed point results in the literature.
\end{abstract}

Keywords: d-metric space, Common fixed point, Weakly compatible maps, Cauchy sequence.

\section{Introduction}

The fixed point theory has become a part of non-linear functional analysis since 1960. It serves as an essential tool to get the solution of the functional relations of various branches of mathematical analysis and its applications. In 1922, Polish mathematician S. Banach [1] established a fixed point theorem in metric space. Since then, a number of fixed point theorems have been proved by many authors and various generalizations of this theorem have been established. In 1986, S.G. Matthews [10] introduced the concept of dislocated metric space under the name of metric domains in domain theory. In 2000, P. Hitzler and A. K. Seda 3 introduced the concept of dislocated topology and generalized the famous Banach Contraction Principle in dislocated metric space. Since then, many authors have established fixed point theorems in dislocated metric space. In the literature one can find many interesting recent articles in the field of dislocated metric space (See for examples [7]- [17]). The study of dislocated metric plays very important role in topology, logic programming and in electronics engineering.

The purpose of this article is to establish a Meir- Keeler type common fixed point theorem for two pairs of weakly compatible mappings in dislocated metric space which improve and extend similar fixed point results in the literature.

\section{Preliminaries}

We start with the following definitions, lemmas and theorems.

Definition 1. [3] Let $X$ be a non empty set and let $d: X \times X \rightarrow[0, \infty)$ be a function satisfying the following conditions:

1. $d(x, y)=d(y, x)$

2. $d(x, y)=d(y, x)=0$ implies $x=y$.

3. $d(x, y) \leq d(x, z)+d(z, y)$ for all $x, y, z \in X$.

Then $d$ is called dislocated metric (or d-metric) on $X$ and the pair $(X, d)$ is called the dislocated metric space (or d-metric space).

Definition 2. 3] A sequence $\left\{x_{n}\right\}$ in a d-metric space $(X, d)$ is called a Cauchy sequence if for given $\epsilon>$ 0 , there corresponds $n_{0} \in N$ such that for all $m, n \geq n_{0}$, we have $d\left(x_{m}, x_{n}\right)<\epsilon$.

Definition 3. [3] A sequence in d-metric space converges with respect to $d$ (or in $d$ ) if there exists $x \in X$ such that $d\left(x_{n}, x\right) \rightarrow 0$ as $n \rightarrow \infty$.

Definition 4. [3] A d-metric space $(X, d)$ is called complete if every Cauchy sequence in it is convergent with respect to $d$. 
Lemma 1. 14 Let $(X, d)$ be a dislocated metric space. Let $A, B, S, T: X \rightarrow X$ be mappings satisfying the conditions

$$
A(X) \subseteq T(X) \text { and } B(X) \subseteq S(X)
$$

Assume further that given for each $\varepsilon>0$ there exists $\delta>0$ such that for all $x, y \in X$

$$
\varepsilon \leq M(x, y)<\varepsilon+\delta \Longrightarrow d(A x, B y) \leq \varepsilon
$$

and

$$
d(A x, B y)<M(x, y) \quad \text { whenever } \quad M(x, y)>0
$$

where

$$
M(x, y)=\max \left\{d(S x, T y), d(A x, S x), d(B y, T y), \frac{1}{2} d(S x, B y), \frac{1}{2} d(A x, T y)\right\}
$$

then for each $x_{0} \in X$, the sequence $\left\{y_{n}\right\}$ in $X$ defined by the rule

$$
y_{2 n}=A x_{2 n}=T x_{2 n+1}, \quad y_{2 n+1}=B x_{2 n+1}=S x_{2 n+2}
$$

is a Cauchy sequence.

Definition 5. [11] A self mapping $T$ of a metric space $(X, d)$ is called a weakly uniformly strict contraction or simply an $(\varepsilon-\delta)$ contraction if for each $\varepsilon>0$ there exists $\delta>0$ such that for all $x, y \in X$

$$
\varepsilon \leq d(x, y)<\varepsilon+\delta \Longrightarrow d(T x, T y)<\varepsilon
$$

Theorem 1. [11] Let $(X, d)$ be a complete metric space and $T: X \rightarrow X$ is weakly uniformly strict contraction then $T$ has a unique common fixed point, say $z$ and for any $x \in X, \quad \lim _{n \rightarrow \infty} T^{n} x=z$.

Definition 6. [6] Let $A$ and $S$ be mappings from a metric space $(X, d)$ into itself. Then, $A$ and $S$ are said to be weakly compatible if they commute at their coincident point; that is, $A x=S x$ for some $x \in X$ implies $A S x=S A x$.

There exists a vast literature which generalizes the result of Meir and Keeler. In 99 Maiti and Pal established a fixed point theorem for a self map $\mathrm{T}$ of a metric space satisfying the following condition which is the generalization of weakly uniformly strict contraction .

For every $\varepsilon>0$ there exists a $\delta>0$ such that

$$
\varepsilon \leq \max \{d(x, y), d(x, T y), d(y, T y)\}<\varepsilon+\delta \quad \Longrightarrow d(T x, T y)<\varepsilon
$$

Park-Rhoades [18] and Rao- Rao [19] extended this result for two self mapping $S$ and $T$ in a metric space $(X, d)$ satisfying the condition

$$
\begin{aligned}
& \varepsilon \leq \max \left\{d(S x, S y), d(S x, T x), d(S y, T y), \frac{1}{2}[d(S x, T y)+d(S y, T x)]\right\}<\varepsilon+\delta \\
& \Longrightarrow d(T x, T y)<\varepsilon
\end{aligned}
$$

In 1986, Jungck [5] and Pant [12] extended the results for four mappings.

\section{Main Results}

Now we establish a common fixed point theorem for two pairs of weakly compatible mappings in dislocated metric space. 
Theorem 2. Let $(X, d)$ be complete dislocated metric space. Let $A, B, S, T: X \rightarrow X$. Let the pairs (A, S) and $(B, T)$ are weakly compatible mappings satisfying the conditions

$$
\begin{aligned}
& A(X) \subseteq T(X) \text { and } B(X) \subseteq S(X) \\
& \text { One of } A(X), B(X), S(X) \text { or } \quad T(X) \text { is closed } \\
& \text { for each } \varepsilon>0 \text {, there exists } \delta>0 \text { such that } \\
& \varepsilon<M(x, y)<\varepsilon+\delta \Longrightarrow d(A x, B y) \leq \varepsilon \\
& x, y \in X, M(x, y)>0 \Longrightarrow d(A x, B y)<M(x, y) \text { where } \\
& M(x, y)=\max \left\{d(S x, T y), d(A x, S x), d(B y, T y), \frac{1}{2} d(S x, B y), \frac{1}{2} d(A x, T y)\right\}
\end{aligned}
$$

and,

$$
\begin{aligned}
d(A x, B y) & \leq k\left[\frac{d(S x, T y) d(S x, A x)}{d(S x, B y)}+d(S x, T y)+d(A x, S x)\right. \\
& \left.+d(B y, T y)+d(S x, B y)+d(A x, T y)+\frac{d(B y, T y) d(A x, T y)}{d(S x, B y)}\right]
\end{aligned}
$$

for $0 \leq k<\frac{1}{11}$. Then $A, B, S$ and $T$ have a unique common fixed point in $X$.

Proof. let $x_{0} \in X$, then by (5) we can define a sequence $\left\{y_{n}\right\}$ in $\mathrm{X}$ such that

$$
y_{2 n}=A x_{2 n}=T x_{2 n+1} \quad \text { and } \quad y_{2 n+1}=B x_{2 n+1}=S x_{2 n+2}, \quad n \in \mathbb{N} \cup\{0\}
$$

Then by the lemma the sequence $\left\{y_{n}\right\}$ is a Cauchy sequence in X.

Since $\mathrm{X}$ is complete, $\left\{y_{n}\right\}$ and its subsequences

$$
\left\{A x_{2 n}\right\}=\left\{T x_{2 n+1}\right\},\left\{S x_{2 n}\right\}=\left\{B x_{2 n-1}\right\} \quad \text { and } \quad\left\{S x_{2 n+2}\right\}=\left\{B x_{2 n+1}\right\}
$$

all converge to a point $z \in X$. Assume that $\mathrm{A}(\mathrm{X})$ is closed, Since $A(X) \subseteq T(X)$ then there exists a point $u \in X$ such that $z=T u$. By the inequality $(9)$ we have

$$
\begin{aligned}
d\left(A x_{2 n}, B u\right) & \leq k\left[\frac{d\left(S x_{2 n}, T u\right) d\left(S x_{2 n}, A x_{2 n}\right)}{d\left(S x_{2 n}, B u\right)}+d\left(S x_{2 n}, T u\right)+d\left(A x_{2 n}, S x_{2 n}\right)\right. \\
& \left.+d(B u, T u)+d\left(S x_{2 n}, B u\right)+d\left(A x_{2 n}, T u\right)+\frac{d(B u, T u) d\left(A x_{2 n}, T u\right)}{d\left(S x_{2 n}, B u\right)}\right]
\end{aligned}
$$

Now taking limit as $n \rightarrow \infty$, we have

$$
d(z, B u) \leq d(B u, z)+d(z, B u) \leq 2 k d(z, B u)
$$

which is a contradiction. Hence, $d(z, B u)=0 \Longrightarrow z=B u$. Thus we have $z=T u=B u$. Since the pair $(\mathrm{B}, \mathrm{T})$ is weakly compatible, so

$$
B T u=T B u \Longrightarrow B z=B T u=T B u=T z
$$

Now we show that $z$ is a fixed point of $\mathrm{B}$. If possible suppose $z \neq B z$, now by condition $(9)$ we have

$$
\begin{aligned}
d\left(A x_{2 n}, B z\right) & \leq k\left[\frac{d\left(S x_{2 n}, T z\right) d\left(S x_{2 n}, A x_{2 n}\right)}{d\left(S x_{2 n}, B z\right)}+d\left(S x_{2 n}, T z\right)+d\left(A x_{2 n}, S x_{2 n}\right)\right. \\
& \left.+d(B z, T z)+d\left(S x_{2 n}, B z\right)+d\left(A x_{2 n}, T z\right)+\frac{d(B z, T z) d\left(A x_{2 n}, T z\right)}{d\left(S x_{2 n}, B z\right)}\right]
\end{aligned}
$$


Now taking limit as $n \rightarrow \infty$ we obtain

$$
\begin{aligned}
d(z, B z) & \leq k\left[d(z, T z)+d(B z, T z)+d(z, B z)+d(z, T z)+\frac{d(B z, T z) d(z, T z)}{d(z, B z)}\right] \\
& =k[d(z, B z)+d(B z, B z)+d(z, B z)+d(z, B z)+d(B z, B z)] \\
& =[3 d(z, B z)+2 d(B z, B z)] \\
& \leq 7 k d(z, B z)
\end{aligned}
$$

which is a contradiction. So, $d(z, B z)=0 \Longrightarrow z=B z$. Hence $z=B z=T z$

Since, $B(X) \subseteq S(X)$ there exists a point $v \in X$ such that $z=S v$

Now by condition 9 we have,

$$
\begin{aligned}
d(A v, B z) \leq & k\left[\frac{d(S v, T z) d(S v, A v)}{d(S v, B z)}+d(S v, T z)+d(A v, S v)\right. \\
+ & \left.d(B z, T z)+d(S v, B z)+d(A v, T z)+\frac{d(B z, T z) d(A v, T z)}{d(S v, B z)}\right] \\
d(A v, z) & \leq k\left[\frac{d(z, z) d(z, A v)}{d(z, z)}+d(z, z)+d(A v, z)\right. \\
& \left.+d(z, z)+d(z, z)+d(A v, z)+\frac{d(z, z) d(A v, z)}{d(z, z)}\right] \\
& =k[4 d(z, A v)+3 d(z, z)] \\
& \leq 10 k d(z, A v)
\end{aligned}
$$

which is a contradiction, thus $d(A v, z)=0 \Longrightarrow A v=z$. hence $A v=S v=z$ Since pair $(\mathrm{A}, \mathrm{S})$ is weakly compatible, so

$$
S A v=A S v \Longrightarrow S z=A z .
$$

Now we claim that $z$ is the fixed point of $A$. For this by condition 90 we have,

$$
\begin{aligned}
d(A z, B z) \leq & k\left[\frac{d(S z, T z) d(S z, A z)}{d(S z, B z)}+d(S z, T z)+d(A z, S z)\right. \\
& \left.+d(B z, T z)+d(S z, B z)+d(A z, T z)+\frac{d(B z, T z) d(A z, T z)}{d(S z, B z)}\right] \\
& =k\left[\frac{d(A z, z) d(A z, A z)}{d(A z, z)}+d(A z, z)+d(A z, A z)\right. \\
& \left.+d(z, z)+d(A z, z)+d(A z, z)+\frac{d(z, z) d(A z, z)}{d(A z, z)}\right] \\
& \\
d(A z, z) & \leq k[2 d(A z, A z)+3 d(A z, z)+2 d(z, z)] \\
& \leq k[4 d(A z, z)+3 d(A z, z)+4 d(A z, z)] \\
& =11 k d(A z, z)
\end{aligned}
$$

which is a contradiction. Consequently we have, $A z=z=S z$. Hence,

$$
A z=B z=S z=T z=z
$$

This represents that $z$ is the common fixed point of the mappings $A, B, S$ and $T$.

\section{Uniqueness:}

If possible, let $z$ and $w$ are two common fixed points of the mappings $A, B, S$ and $T$. Now by condition (9) 
we have,

$$
\begin{aligned}
d(A z, B w) \leq & k\left[\frac{d(S z, T w) d(S z, A z)}{d(S z, B w)}+d(S z, T w)+d(A z, S z)\right. \\
+ & \left.d(B w, T w)+d(S z, B w)+d(A z, T w)+\frac{d(B w, T w) d(A z, T w)}{d(S z, B w)}\right] \\
= & k\left[\frac{d(z, w) d(z, z)}{d(z, w)}+d(z, w)+d(z, z)\right. \\
+ & \left.d(w, w)+d(z, w)+d(z, w)+\frac{d(w, w) d(z, w)}{d(z, w)}\right] \\
& \\
& \quad \begin{aligned}
& d(z, w) \\
& \leq k[2 d(z, z)+3 d(z, w)+2 d(w, w)] \\
& \leq \\
&=11 k d(z, w)
\end{aligned}
\end{aligned}
$$

which is a contradiction. Hence, $d(z, w)=0 \Longrightarrow z=w$. This completes the proof of the theorem.

Similarly, we can establish the conclusion by supposing $\mathrm{B}(\mathrm{X})$ (resp. S(X), T(X)) is closed.

Now with the light of above theorem, one can easily establish the following corollaries:

Corrollary 1. Let $(X, d)$ be complete dislocated metric space. Let $A, B, S: X \rightarrow X$. Let the pairs (A, S) and $(B, S)$ are weakly compatible mappings satisfying the conditions

$$
\begin{gathered}
A(X), B(X) \subseteq S(X) \\
\text { One of } A(X), B(X) \quad \text { or } \quad S(X) \quad \text { is closed } \\
\text { for each } \varepsilon>0, \quad \text { there exists } \delta>0 \quad \text { such that } \\
\varepsilon<M(x, y)<\varepsilon+\delta \Longrightarrow d(A x, B y) \leq \varepsilon \\
x, y \in X, M(x, y)>0 \Longrightarrow d(A x, B y)<M(x, y) \quad \text { where } \\
M(x, y)=\max \left\{d(S x, S y), d(A x, S x), d(B y, S y), \frac{1}{2} d(S x, B y), \frac{1}{2} d(\text { Ax, Sy })\right\}
\end{gathered}
$$

and

$$
\begin{aligned}
d(A x, B y) & \leq k\left[\frac{d(S x, S y) d(S x, A x)}{d(S x, B y)}+d(S x, S y)+d(A x, S x)\right. \\
& \left.+d(B y, S y)+d(S x, B y)+d(A x, S y)+\frac{d(B y, S y) d(A x, S y)}{d(S x, B y)}\right]
\end{aligned}
$$

for $0 \leq k<\frac{1}{11}$. Then $A, B$ and $S$ have a unique common fixed point in $X$.

Corrollary 2. Let $(X, d)$ be complete dislocated metric space. Let $A, S, T: X \rightarrow X$. Let the pairs (A, S) and $(A, T)$ are weakly compatible mappings satisfying the conditions

$$
A(X) \subseteq T(X) \text { and } S(X)
$$

One of $A(X), S(X)$ or $T(X)$ is closed

$$
\begin{array}{r}
\text { for each } \varepsilon>0, \text { there exists } \delta>0 \quad \text { such that } \\
\varepsilon<M(x, y)<\varepsilon+\delta \Longrightarrow d(A x, A y) \leq \varepsilon
\end{array}
$$




$$
\begin{array}{r}
x, y \in X, M(x, y)>0 \Longrightarrow d(A x, A y)<M(x, y) \quad \text { where } \\
M(x, y)=\max \left\{d(S x, T y), d(A x, S x), d(A y, T y), \frac{1}{2} d(S x, A y), \frac{1}{2} d(A x, T y)\right\}
\end{array}
$$

and

$$
\begin{aligned}
d(A x, A y) & \leq k\left[\frac{d(S x, T y) d(S x, A x)}{d(S x, A y)}+d(S x, T y)+d(A x, S x)\right. \\
& \left.+d(A y, T y)+d(S x, A y)+d(A x, T y)+\frac{d(A y, T y) d(A x, T y)}{d(S x, A y)}\right]
\end{aligned}
$$

for $0 \leq k<\frac{1}{11}$. Then $A, S$ and $T$ have a unique common fixed point in $X$.

Corrollary 3. Let $(X, d)$ be complete dislocated metric space. Let $A, S: X \rightarrow X$. Let the pair $(A, S)$ is weakly compatible mappings satisfying the conditions

$$
\begin{gathered}
A(X) \subseteq S(X) \\
\text { One of } A(X) \text { or } S(X) \quad \text { is closed } \\
\text { for each } \varepsilon>0, \quad \text { there exists } \delta>0 \quad \text { such that } \\
\varepsilon<M(x, y)<\varepsilon+\delta \Longrightarrow d(A x, A y) \leq \varepsilon \\
x, y \in X, M(x, y)>0 \Longrightarrow d(A x, A y)<M(x, y) \text { where } \\
M(x, y)=\max \left\{d(S x, S y), d(A x, S x), d(\text { Ay, Sy }), \frac{1}{2} d(S x, A y), \frac{1}{2} d(\text { Ax, Sy })\right\}
\end{gathered}
$$

and

$$
\begin{aligned}
d(A x, A y) & \leq k\left[\frac{d(S x, S y) d(S x, A x)}{d(S x, A y)}+d(S x, S y)+d(A x, S x)\right. \\
& \left.+d(A y, S y)+d(S x, A y)+d(A x, S y)+\frac{d(A y, S y) d(A x, S y)}{d(S x, A y)}\right]
\end{aligned}
$$

for $0 \leq k<\frac{1}{11}$. Then $A$ and $S$ have a unique common fixed point in $X$.

\section{Conclusion}

Our result generalizes the Meir- Keeler [11] fixed point theorem in dislocated metric space and extends the results of Pant and Jha [13], Bouhadjera and Djoudi [2], Jha, Pant and Singh [4] and Panthi [14.

\section{Acknowledgement}

This work is carried out under Small Research Development and Innovation Grant SRDIG-73/74-S\&T-08 supported by University Grants Commission, Nepal.

\section{References}

[1] Banach S (1922) Sur les operations dans les ensembles abstraits et leur applications aux equations integrales, fundamental Mathematicae,3(7), 133-181.

[2] Bouhadjera H and Djoudi A (2008) On common fixed points of Meir and Keeler type, An. St. Univ. Ovidlus Constanta, 16(2), 39-46. 
[3] Hitzler P and Seda A K (2000) Dislocated Topologies, J. Electr. Engg., 51 (12/s), 3-7.

[4] Jha K, Pant RP and Singh SL (2003) Common fixed points for compatible mappings in metric space, Rad. Mat.12, 107-114.

[5] Jungck G (1986) Compatible mappings and common fixed points, Int. J. Math. Sci., 9, 771-779.

[6] Jungck G and Rhoades BE (1998) Fixed points for set valued functions without continuity, Indian J. Pure Appl. Math.. 29(3), 227-238.

[7] Kumari PS, Zoto K, and Panthi D (2015) d-Neighborhood system and generalized F-contraction in dislocated metric space, SpringerPlus, 4(1), 1-10.

[8] Kumari PS, Ramana CV, Zoto K, and Panthi D (2015) Fixed point theorems and generalizations of dislocated metric spaces, Indian J. Sci. Tech. 8(S3), 154-158.

[9] Maiti M and Pal TK (1978) Generalization of two fixed-point theorems, Bull. Calcutta Math. Soc., 70, 57-61.

[10] Matthews SG (1986) Metric domains for completeness. Technical report 76 [Ph.D. Thesis], Department of Computer Science, University of Warwick, Coventry, UK.

[11] Meir A and Keeler E (1969) A theorem in contraction mappings, J. Math. Anal. Appl. ,28, 326-329.

[12] Pant RP (1986) Common fixed point of two pair of commuting mappings, Indian J. Pure Appl. Math. 17(2), 187- 192 .

[13] Pant RP and Jha K (2002) A generalization of Meir- Keeler type common fixed point theorem for four mappings, J. Nat. Phys. Sci., 16(1-2), 77-84.

[14] Panthi D (2018) Some Common Fixed Point Theorems Satisfying Meir-Keeler Type Contractive Conditions, Open J. Disc.Math., 8(2), 35-47.

[15] Panthi D (2015) Common fixed point theorems for compatible mappings in dislocated metric space. Int. J. Math. Anal., 9(45), 2235-2242.

[16] Panthi D and Kumari PS (2016) Some Integral Type Fixed Point Theorems in Dislocated Metric Space, American. J. Comp. Math,6, 88-97.

[17] Panthi D and Subedi K (2016) Some Common Fixed Point Theorems for Four Mappings in Dislocated Metric Space, Advances in pure mathematics, 6, 695-712

[18] Park S and Rhoades BE (1981) Meir- Keeler type contractive conditions, Math. Japonica, 26 (1) 13-20.

[19] Rao IHN and Rao KPR (1985) Generalization of fixed-point theorems of Meir - Keleer type, Indian J. Pure Appl. Math., 16, 1249-1262. 